\title{
Increased Serum Levels of Tissue Inhibitor of Metalloproteinase-1 in Patients With Acute Myocardial Infarction
}

\author{
Wilfried Dinh, ${ }^{1,2^{*}}$ MD, Reiner FüTH, ${ }^{1,2^{*}}$ MD, Thomas ScheFFOLD, ${ }^{2}$ MD, \\ Lars BANSEMIR, ${ }^{1,2}$ MD, Till KöHLER, ${ }^{1}$ MD, Harald LAPP, ${ }^{3}$ MD, \\ Alexander BufE, ${ }^{1} \mathrm{MD}$, Werner NICKL, ${ }^{1,2} \mathrm{MD}$, Johannes-Peter $\mathrm{STASCH},{ }^{4} \mathrm{PhD}$, \\ and Mark LANKISCH, ${ }^{1,2} \mathrm{MD}$
}

\section{SUMMARY}

It has been suggested that matrix-metalloproteinases (MMPs) and their inhibitors (tissue inhibitors of metalloproteinases (TIMPs) play a major role in the regulation of myocardial remodeling. Myocardial extracellular matrix (ECM) is highly susceptible to ischemic injury in acute myocardial infarction (AMI).

We measured serum levels of TIMP-1 in the early hours of AMI to study the kinetics of these enzymes in an early ischemic phase.

TIMP-1 was measured in 25 patients with AMI and 116 healthy controls. Blood samples were obtained during the first 12 hours after hospital admission. Left ventricular function (LVF) and hemodynamic data were collected during coronary intervention.

TIMP-1 was significantly elevated in patients with AMI within the first hours compared to controls $(P<0.05)$. No significant difference was observed between patients with preserved LVF and with impaired LVF. Elevated TIMP-1 levels did not correlate with increased levels of CK or CK-MB band during the first hours after AMI.

Increased TIMP-1 can be detected within 12 hours in patients with AMI, suggesting early onset of remodeling. Elevation of TIMP-1 may be a surrogate marker for increased ECM-turnover. The prognostic relevance needs to be proved in long-term studies. (Int Heart J 2009; 50: 421-431)

Key words: Biomarkers, Remodeling, Extracellular matrix, TIMP-1, Acute myocardial infarction

IN myocardial tissue the myocytes are embedded in a fibrillar network of collagen responsible for normal myocardial structure and function. ${ }^{1-3)}$ Hemodynamic

From the ${ }^{1}$ Helios Clinics Wuppertal, Department of Cardiology, Wuppertal, ${ }^{2}$ Institute for Heart and Circulation Research, University Witten Herdecke, Witten, ${ }^{3}$ Helios Clinics Erfurt, Heart Center, Erfurt, ${ }^{4}$ Cardiovascular Research, Bayer HealthCare, Wuppertal, Germany.

*Equal contribution

Address for correspondence: Mark Lankisch, MD, Helios Clinics Wuppertal, Department of Cardiology, Arrenberger Strasse 20, D-42117 Wuppertal, Germany.

Received for publication October 31, 2008

Revised and accepted February 5, 2009. 
pressure or volume load and ischemic injury lead to structural myocardial modifications which are reflected in changes of myocyte mass and changes in the production and composition of proteins of the extracellular matrix (ECM). ${ }^{4-6)}$ Left ventricular (LV) remodeling is an important contributory event in the progression of congestive heart failure after myocardial infarction. ${ }^{4,6-8)}$ Serum levels of circulating proteins of the ECM-turnover may be helpful to identify and quantify these structural changes. ${ }^{9-11)}$ Endogenous enzymes responsible for extracellular collagen degradation and remodeling are the family of matrix-metalloproteinases (MMPs). MMPs are enzymes capable of degrading all of the major components of the extracellular matrix and are thought to have an important function in tissue repair and remodeling. Regulation of these enzymes occurs at several levels including gene expression and secretion as latent precursor enzymes that have to be activated in the extracellular space. A final level of regulation occurs through inhibition of the enzyme activity by alpha 2-macroglobulin or more specifically by tissue inhibitors of metalloproteinases (TIMP). There are at least 4 different types of TIMP (TIMP-1, 2, 3 and 4). TIMP-1 is a glycoprotein of $28.5 \mathrm{kD}$ that inhibits all MMPs by forming tight and noncovalent complexes with the activated enzymes in a stochiometric fashion. MMP is also able to bind TIMP-1 in its precursor form. A second, more recently characterised inhibitor is TIMP-2, a nonglycosylated molecule of $21.5 \mathrm{kD}$ that shares $41 \%$ sequence homology with TIMP-1. However, TIMP-1 is more widely distributed or at least better characterised than other TIMPs. TIMP-1 is secreted by a wide variety of fibroblasts and other cell types. In addition to its function as a regulator of MMP activity, TIMP-1 appears to have other physiological functions as well. An increase in TIMP-1 within the acute phase of myocardial infarction may indicate the early activation of the MMP/TIMP system in the infarcted myocardium. The degrading effects of activated MMP-1 on ECM are inhibited by TIMP-1. Therefore, increased levels of TIMP-1 in the systemic circulation may reflect the increased production of these enzymes and their release from the infarcted myocardium.

The aim of the present study was to investigate if these biomarkers are increased immediately after the onset of AMI and may therefore be useful to identify patients at high risk for developing congestive heart failure.

\section{Methods}

Patients and protocol: We prospectively studied 25 consecutive patients (22 men and 3 women, mean age, 58 years, range, 43 to 78 years) admitted to our hospital with an acute myocardial infarction (AMI). The investigation conformed with the principles outlined in the Declaration of Helsinki. Myocardial infarction was diagnosed on the basis of at least two of the following criteria: (1) prolonged 
typical chest pain $>30$ minutes, (2) persistent significant electrocardiographic changes in two ore more contiguous leads compatible with myocardial ischemia, and (3) an increase in serum creatine phosphokinase (CPK) with a significant MB band. Exclusion criteria were prior myocardial infarction, significant valvular heart disease, cardiomyopathy, non-Q myocardial infarction, as well as preexisting heart failure and liver disease. Venous blood samples were taken within 12 hours after the onset of AMI, centrifuged, divided into aliquots, and stored at $-80^{\circ} \mathrm{C}$ until use. Clinical, routine laboratory, and invasive data of the patients were prospectively stored in a database $\left(\mathrm{SPSS}^{\circledR}\right)$. All patients underwent cardiac catheterization and, with one exception, primary PTCA.

Laboratory analysis: Measurements of TIMP-1 were performed in serum using an automated immuno assay. The assay was developed for the BAYER IMMUNO $1^{\complement}$ immunoassay system. The TIMP-1 assay is a heterogeneous immunoassay with magnetic particle separation technique. The linear assay range was $0-3000 \mathrm{ng} / \mathrm{mL}$ and the minimum detectable dose of the assay was calculated to be $<2 \mathrm{ng} / \mathrm{mL}$. Recoveries for all samples described were between $98.6 \%$ and $101.8 \%$. The results obtained demonstrated a good linearity over the entire assay range.

Statistical analysis: Statistical analysis was performed with SPSS ${ }^{\circledR}$. The results are expressed as the mean $\pm \mathrm{SD}$. We used the unpaired $t$ test for continuous variables between controls and patients with AMI, and the paired $t$-test was used for within group comparisons. Values of $P<0.05$ were considered statistically significant. Correlation was calculated using the Spearman-rho correlation coefficient.

\section{RESUlts}

Control patients: In order to establish preliminary reference intervals and to investigate TIMP-1 levels for different ages and sex, sera of 116 apparently healthy volunteers without history of heart disease, liver disease, or high blood pressure were tested. Mean TIMP-1 concentrations were $626.8 \pm 119.3 \mathrm{ng} / \mathrm{mL}$ (mean $\pm \mathrm{SD}$ ). The stratification of data according to sex or age subgroups was not necessary. Reference range data for TIMP-1 are demonstrated in Table I.

Study group: Twenty-five patients with a first acute myocardial infarction were included. Table II shows the clinical characteristics and results from cardiac catheterization in the setting of primary percutaneus transluminal coronary angioplasty (PTCA), excluding one patient without PTCA. The mean values of CPK (U/L) were 1037, SD \pm 668 , range, 239 to 2646, and the mean values of $\mathrm{CK}$ $\mathrm{MB}(\mathrm{U} / \mathrm{L})$ were $100.90(\mathrm{SD} \pm 65.14)$.

Serum levels of TIMP-1 and infarct size: Serum levels of TIMP-1 were sig- 
Table I. Serum Levels of TIMP-1 in Healthy Control Subjects

\begin{tabular}{lcc}
\hline & Female $>40$ years & Male $>40$ years \\
\hline$n$ & 62 & 54 \\
\hline Mean $(\mathrm{ng} / \mathrm{mL})$ & 631.7 & 623.7 \\
\hline $\mathrm{SD}(\mathrm{ng} / \mathrm{mL})$ & 103.8 & 135.4 \\
\hline
\end{tabular}

Table II. Clinical Characteristics and Angiographic Results of Study Patients

\begin{tabular}{ll}
\hline Basic characteristics & 25 \\
$n$ & $22 / 3$ \\
Male $(n) /$ Female $(n)$ & $58 \pm 9$ \\
Age (years) & \\
Cardiovascular risk factors & $16 / 25(64 \%)$ \\
Hypertension & $5 / 25(20 \%)$ \\
Diabetes & $19 / 25(76 \%)$ \\
Smoking & 21 \\
Hypercholesterolemia & $5 / 25(20 \%)$ \\
Family history of CAD & \\
Cardiac catheterization & $12 / 25(48 \%)$ \\
EF ${ }^{*}>60 \%$ & $12 / 25(48 \%)$ \\
EF ${ }^{*} 40-60 \%$ & $1 / 25(4.0 \%)$ \\
EF ${ }^{*}<40 \%$ & $15 / 25(60 \%)$ \\
Single vessel disease & $10 / 25(40 \%)$ \\
Multivessel disease & 22 \\
Anterior myocardial infarction & 3 \\
Posterior myocardial infarction & \\
Medication on admission & \\
ACE-inhibitors & $4 / 25(18 \%)$ \\
$\beta$-blockers & $6 / 16(37 \%)$, in 9 cases no data available \\
\hline
\end{tabular}

*EF denotes ejection fraction and CAD coronary artery disease.

nificantly higher in patients with acute myocardial infarction than in control subjects $(702.3 \pm 262.5$ versus $626.8 \pm 119.3, P<0.05$, respectively) as shown in Figure 1. We could not demonstrate a statistically significant correlation between serum levels of TIMP-1 and the maximal creatine-phosphokinase levels $(r=0.16, P=0.5)$ or CK-MB- levels $(r=0.12, P=0.6)$. Also, there was no significant difference between TIMP-1 levels and left ventricular ejection fraction $(P$ $=0.2)$ (Figure 2).

Serum levels of TIMP-1 and clinical characteristics: Gender did not affect TIMP-1 serum levels $(P=0.6)$. There was no statistically significant difference between patients with or without baseline treatment with ACE-inhibitors or $\beta$-blockers before admission to hospital. In addition, there was no significant difference between patients with single or multiple vessel disease $(P=0.32)$ or patients with posterior or anterior myocardial infarction.

Mean TIMP serum level was $804.4 \mathrm{ng} / \mathrm{mL}$ in 16 patients with a history of 


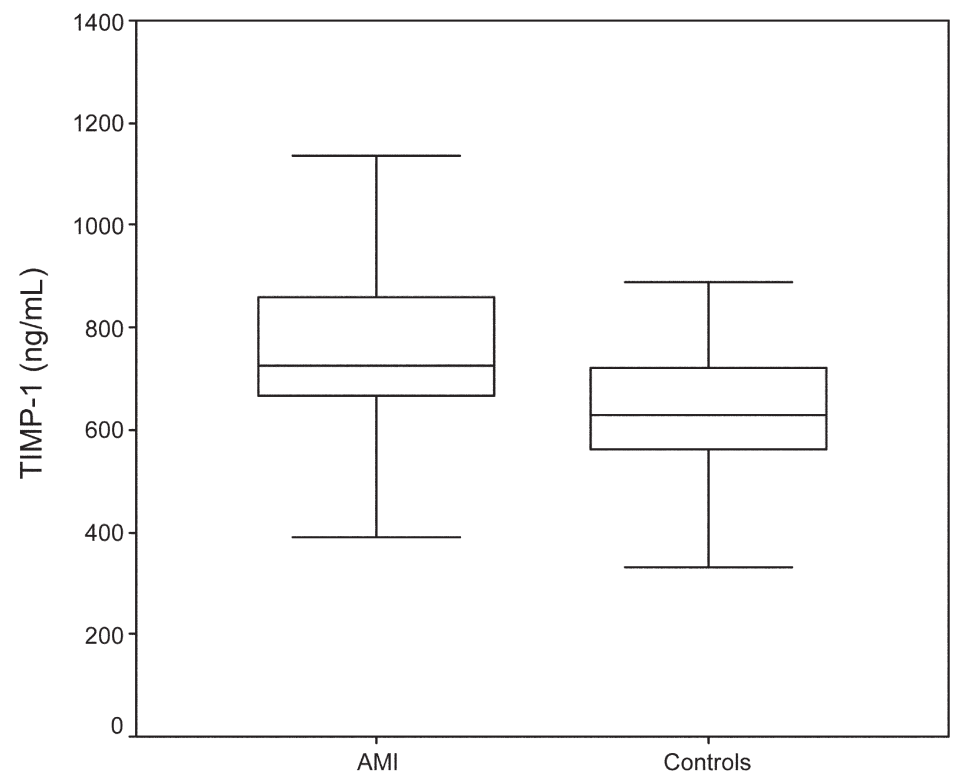

Figure 1. Serum levels of TIMP-1 in controls and patients with acute myocardial infarction (AMI).

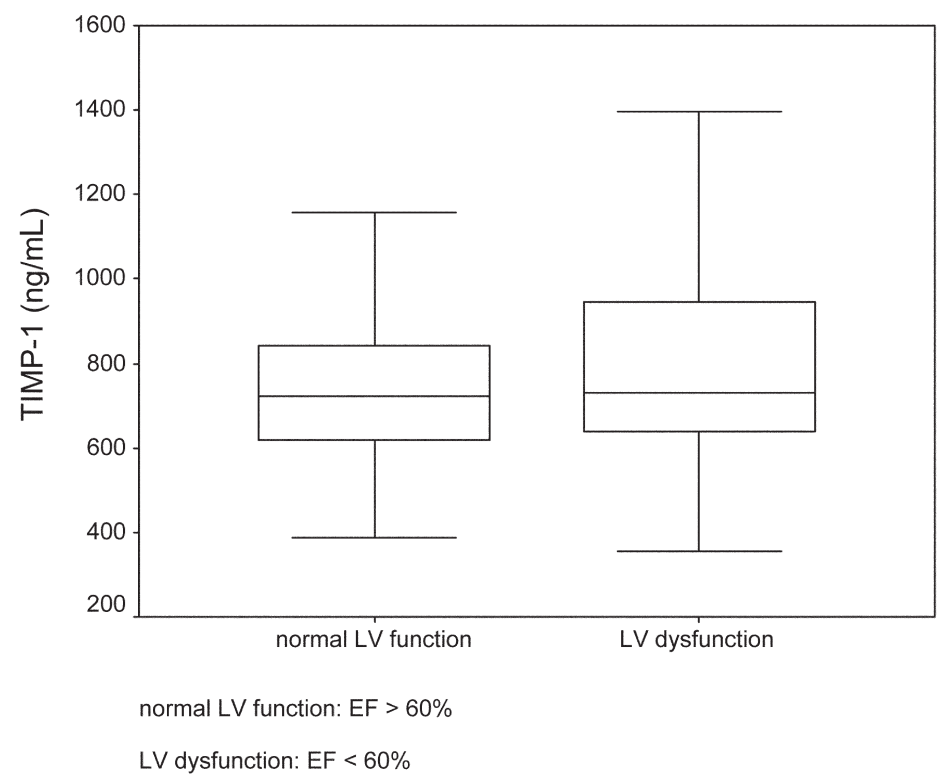

Figure 2. Serum levels of TIMP-1 in patients with normal left ventricular function and reduced left ventricular (LV) function. 
high blood pressure and $659.4 \mathrm{ng} / \mathrm{mL}$ in 9 normotensive subjects. Mean serum level was $880.4 \mathrm{ng} / \mathrm{mL}$ (3 cases) in patients with atrial fibrillation compared to $734.7 \mathrm{ng} / \mathrm{mL}$ (22 cases) in patients with sinus rhythm. Neither the effect of hypertension $(P=0.2)$ nor rhythm reached statistical significance $(P=0.7)$.

\section{Discussion}

We were able to demonstrate that TIMP-1 serum levels are significantly elevated during the first 12 hours after acute myocardial infarction as a possible surrogate marker of early activation extracellular myocardial matrix metabolism. During this early period this rapid activation may represent unspecific repair because it is not correlated to the extent of ischemic damage or other clinical variables. Remodeling starts immediately after coronary artery occlusion and involves degradation of normal ECM, invasion of inflammatory cells at the site of initial injury, and the induction of bioactive peptides and cytokines, which among other things may be responsible for the induction of TIMP-1 in this study. In our study, the induction of TIMP-1 seems to be independent of other markers of inflammation, such as CRP levels.

Myocardial infarction causes complex structural changes in the left ventricular myocardium. TIMPs bind to MMPs in a stoichiometric 1:1 molar ratio and therefore form an important endogenous system for regulation of MMP activity in vivo. ${ }^{12)}$ Several reports have provided evidence to support the concept that increased MMP activity may contribute to the development of LV remodeling and a poor prognosis. ${ }^{11,13-20)}$ Coexpression of MMP and TIMP has been shown in the myocardium. However, whether this is reflected by increased activity of the endogenous inhibitor system TIMP-1 in the serum and to what degree MMP inhibition may influence the LV remodeling process is currently under investigation. How can our findings therefore be interpreted?

The method we used for determining TIMP-1 serum levels is well established. The increased serum levels of TIMPs in the early phase of myocardial infarction can be explained in several ways. We hypothesised that increased serum levels of TIMP-1, which acts like a negative inhibitor of MMPs, reflect the ECM remodeling involved in the process of postischemic tissue degradation and repair. Our findings suggested that remodeling starts very early after ischemic injury and is reflected by increased serum levels of TIMP-1. Therefore, TIMP-1 may be a serological surrogate parameter for left ventricular remodeling after acute myocardial infarction. Inokubo, et al have shown that the great cardiac vein-aortic root difference in serum TIMP-1 and MMP-9 levels in patients with acute coronary syndrome was significantly higher than in control subjects with normal coronary arteries. They also showed increased peripheral serum levels of 
TIMP-1 on day 3 after AMI which lasted up to 3 weeks after onset of AMI. ${ }^{21)} \mathrm{A}$ study by Hirohata, et al also showed comparable results. ${ }^{22)}$ In addition to these findings, we were able to demonstrate that already within the first 12 hours after myocardial infarction the TIMP-1 serum levels increased significantly, as a sign for very early myocardial remodeling.

We demonstrated that there is no significant correlation between TIMP-1 levels and maximal creatine-phosphokinase levels, LV function, or localisation of the infarct during the early ischemic period. It should be taken into account that remodeling starts early after ischemic injury, as reflected in elevated TIMP-1 serum levels, but then may turn into a chronic process. ${ }^{23)}$ The progress of remodeling is influenced by many different factors, such as the MMP/TIMPsystem, cytokines, and neuroendocrine activation. ${ }^{6,24-28)}$ Therefore, the degree of TIMP-1 activation may not only depend on the size of acute damage, but also on other pathophysiological changes starting after AMI. ${ }^{24)}$ As a consequence, the degree of TIMP elevation does not preliminarily mirror the size of acute myocardial damage. Rather, it reflects, as a part of a complex regulator system, the ability to counterbalance the degradation of collagen matrix caused by MMP activation during remodeling. This may be the reason we did not find a significant correlation between serum levels of TIMP-1 to clinical findings (NYHA, Killip), LV diameter, or LV aneurysm formation in the first 12 hours after AMI. Furthermore, ECM structure differs from patient to patient with respect to the number of myocytes and fibroblasts. This may partly account for the lack of a direct significant correlation between the CK levels and changes in TIMP-1.

With respect to the physiological effects of TIMP-1, we postulated that increased TIMP activity might be a protective mechanism to provide high turnover rates in ECM in the early phase of acute myocardial infarction. Measurement of cardiac remodeling using serological surrogate markers may be a useful tool for monitoring cardiac tissue repair in clinical conditions after acute myocardial infarction. ${ }^{9-10,29-32)}$ There is already accumulating evidence to show that serum levels of procollagen peptide fragments and of metalloproteinases can be used as markers for cardiac collagen turnover. ${ }^{29,30,33)}$ Additional studies focusing on the prognostic value of increased TIMP-1 levels in regard to the development of LV dysfunction would be appropriate. The next step may be to investigate whether TIMP and MMP can provide an important therapeutic target for the discovery of new drugs for preventing and treating chronic heart failure after ischemic injury. From the current evidence, it is likely that modulation of MMPs after AMI and in the development of chronic heart failure through factors that affect MMP activity will alter the ECM remodeling process. This will eventually alter the progression in the development of ischemic heart disease after acute myocardial infarction. Spinale, et al tested the hypothesis that interruption of myocardial 
MMP activity will reduce the LV dilatation. The nonselective MMP inhibitor PD166793 was used in a pig model of pacing CHF. It could be shown that the degree of LV dilatation significantly progressed without treatment and was accompanied by reduced pump function and posterior wall thickness. With MMP inhibition, the degree of LV dilatation was attenuated and LV pump function improved. ${ }^{34)}$ Other studies have demonstrated that combined therapy with an angiotensin converting enzyme inhibitor and MMP inhibition reduced LV chamber dimensions in pacing congestive heart failure (CHF). ${ }^{35,36)}$ Rhode, et al demonstrated that MMP inhibition attenuated the LV dilatation which occurred in the early postinfarction period in a myocardial infarction model in mice. ${ }^{37}$

There are a number of limitations in our study that must be recognized. First, because of strict inclusion criteria, a small number of patients were included. Second, this study could not address whether potential changes in the TIMP-1 stoichiometric relation to specific MMPs may have occurred during the development of AMI and in the first hours after onset of AMI. Third, it is also possible that changes in the equilibrium between tissue and serum concentrations may be caused by metabolism or mobilisation from serum to the interstitial spaces. Finally, there is evidence about the influence of cytokines like TNF and IL-1 on TIMP and MMP activation and activity after acute myocardial infarction. $^{38-40)}$

Conclusion: Increased serum levels of TIMP-1 can be detected within 12 hours in patients with a first acute myocardial infarction, suggesting an early onset of myocardial remodeling. We hypothesised that in the early phase of myocardial infarction, this may reflect the ability to prevent a high turnover rate of ECM caused by MMP activation which has been reported to occur with the development of chronic heart failure. Further investigations are needed to examine whether these findings provide significant clinical prognostic information. Regulation of MMP activity by synthetic inhibitors (TIMP- agonists) may be an important therapeutic target for controlling ECM remodeling in the setting of developing heart failure. Other questions, which will need to be further pursued from our studies, include if increased serum levels of TIMP provide prognostic information and how MMP inhibition will be integrated into the pharmacological arsenal of myocardial infarction and CHF. 


\section{ACKNOWLEDGEMENT}

We are grateful to the Cardiovascular Research section of Bayer HealthCare for supporting the study.

\section{REFERENCES}

1. Robinson TF, Cohen-Gould L, Remily RM, Capasso JM, Factor SM. Extracellular structures in heart muscle. Adv Myocardiol 1985; 5: 243-55.

2. Erlebacher JA, Weiss JL, Weisfeldt ML, Bulkley BH. Early dilation of the infarcted segment in acute transmural myocardial infarction: role of infarct expansion in acute left ventricular enlargement. J Am Coll Cardiol 1984; 4: 201-8.

3. Nagase H, Visse R, Murphy G. Structure and function of matrix metalloproteinases and TIMPs. Cardiovasc Res 2006; 69: 562-73.

4. Pfeffer MA, Braunwald E. Ventricular remodeling after myocardial infarction. Experimental observations and clinical implications. Circulation 1990; 81: 1161-72. (Review)

5. Sutton MG, Sharpe N. Left ventricular remodeling after myocardial infarction: pathophysiology and therapy. Circulation 2000; 101: 2981-8. (Review)

6. Swynghedauw B. Molecular mechanisms of myocardial remodeling. Physiol Rev 1999; 79: 215-62. (Review)

7. Pinet F, Beseme O, Cieniewski-Bernard C, et al. Predicting left ventricular remodeling after a first myocardial infarction by plasma proteome analysis. Proteomics 2008; 8: 1798-808.

8. Krzemiński TF, Nozyński JK, Grzyb J, Porc M. Wide-spread myocardial remodeling after acute myocardial infarction in rat. Features for heart failure progression. Vascul Pharmacol 2008; 48: 100-8.

9. Weber KT. Monitoring tissue repair and fibrosis from a distance. Circulation 1997; 96: 2488-92. (Review)

10. Klappacher G, Franzen P, Haab D, et al. Measuring extracellular matrix turnover in the serum of patients with idiopathic or ischemic dilated cardiomyopathy and impact on diagnosis and prognosis. Am J Cardiol 1995; 75: 913-8.

11. Jordán A, Roldán V, García M, et al. Matrix metalloproteinase-1 and its inhibitor, TIMP-1, in systolic heart failure: relation to functional data and prognosis. J Intern Med 2007; 262: 385-92.

12. Nagase H. Activation mechanisms of matrix metalloproteinases. Biol Chem 1997; 378: 151-60. (Review)

13. Takahashi S, Barry AC, Factor SM. Collagen degradation in ischaemic rat hearts. Biochem J 1990; 265: 233-41.

14. Gunja-Smith Z, Morales AR, Romanelli R, Woessner JF Jr. Remodeling of human myocardial collagen in idiopathic dilated cardiomyopathy. Role of metalloproteinases and pyridinoline cross-links. Am J Pathol 1996; 148: 1639-48.

15. Janicki JS, Brower GL, Henegar JR, Wang L. Ventricular remodeling in heart failure: the role of myocardial collagen. Adv Exp Med Biol 1995; 382: 239-45. (Review)

16. Spinale FG, Coker ML, Thomas CV, Walker JD, Mukherje R, Hebber L. Time-dependent changes in matrix metalloproteinase activity and expression during the progression of congestive heart failure: relation to ventricular and myocyte function. Circ Res 1998; 82: 482-95.

17. Coker ML, Thomas CV, Clair MJ, et al. Myocardial matrix metalloproteinase activity and abundance with congestive heart failure. Am J Physiol 1998; 274: H1516-23.

18. Thomas CV, Coker ML, Zellner JL, Handy JR, Crumbley AJ 3rd, Spinale FG. Increased matrix metalloproteinase activity and selective upregulation in LV myocardium from patients with end-stage dilated cardiomyopathy. Circulation 1998; 97: 1708-15.

19. Elmas E, Lang S, Erik Dempfle CE, et al. High plasma levels of tissue inhibitor of metalloproteinase-1 (TIMP-1) and interleukin-8 (IL-8) characterize patients prone to ventricular fibrillation complicating myocardial infarction. Clin Chem Lab Med 2007; 45: 1360-5. 
20. Vanhoutte D, Schellings M, Pinto Y, Heymans S. Relevance of matrix metalloproteinases and their inhibitors after myocardial infarction: a temporal and spatial window. Cardiovasc Res 2006; 69: 604-13. (Review)

21. Inokubo Y, Hanada H, Ishizaka H, Fukushi T, Kamada T, Okumura K. Plasma levels of matrix metalloproteinase- 9 and tissue inhibitor of metalloproteinase- 1 are increased in the coronary circulation in patients with acute coronary syndrome. Am Heart J 2001; 141: 211-7.

22. Hirohata S, Kusachi S, Murakami M, et al. Time dependent alterations of serum matrix metalloproteinase- 1 and metalloproteinase- 1 tissue inhibitor after successful reperfusion of acute myocardial infarction. Heart 1997; 78: 278-84.

23. Beltrami CA, Finato N, Rocco M, et al. Structural basis of end-stage failure in ischemic cardiomyopathy in humans. Circulation 1994; 89: 151-63.

24. Cleutjens JP, Kandala JC, Guarda E, Guntaka RV, Weber KT. Regulation of collagen degradation in the rat myocardium after infarction. J Mol Cell Cardiol 1995; 27: 1281-92.

25. Dollery CM, McEwan JR, Henney AM. Matrix metalloproteinases and cardiovascular disease. Circ Res 1995; 77: 863-8. (Review)

26. Rouleau JL, de Champlain J, Klein M, et al. Activation of neurohumoral systems in postinfarction left ventricular dysfunction. J Am Coll Cardiol 1993; 22: 390-8.

27. Ikonomidis JS, Hendrick JW, Parkhurst AM, et al. Accelerated LV remodeling after myocardial infarction in TIMP-1-deficient mice: effects of exogenous MMP inhibition. Am J Physiol Heart Circ Physiol 2005; 288: H149-58.

28. Trescher K, Bernecker O, Fellner B, et al. Inflammation and postinfarct remodeling: overexpression of IkappaB prevents ventricular dilation via increasing TIMP levels. Cardiovasc Res 2006; 69: 746-54.

29. Uusimaa P, Risteli J, Niemelä M, et al. Collagen scar formation after acute myocardial infarction: relationships to infarct size, left ventricular function, and coronary artery patency. Circulation 1997; 96 : 2565-72.

30. Cavusoglu E, Ruwende C, Chopra V, et al. Tissue inhibitor of metalloproteinase-1 (TIMP-1) is an independent predictor of all-cause mortality, cardiac mortality, and myocardial infarction. Am Heart J 2006; 151: 1101. e1-8.

31. Lubos E, Schnabel R, Rupprecht HJ, et al. Prognostic value of tissue inhibitor of metalloproteinase-1 for cardiovascular death among patients with cardiovascular disease: results from the AtheroGene study. Eur Heart J 2006; 27: 150-6.

32. Kelly D, Khan SQ, Thompson M, et al. Plasma tissue inhibitor of metalloproteinase-1 and matrix metalloproteinase-9: novel indicators of left ventricular remodelling and prognosis after acute myocardial infarction. Eur Heart J 2008.

33. Sato Y, Kataoka K, Matsumori A, et al. Measuring serum aminoterminal type III procollagen peptide, 7S domain of type IV collagen, and cardiac troponin $\mathrm{T}$ in patients with idiopathic dilated cardiomyopathy and secondary cardiomyopathy. Heart 1997; 78: 505-8.

34. Spinale FG, Coker ML, Krombach SR, et al. Matrix metalloproteinase inhibition during the development of congestive heart failure: effects on left ventricular dimensions and function. Circ Res 1999; 85: $364-76$.

35. McElmurray JH 3rd, Mukherjee R, New RB, et al. Angiotensin-converting enzyme and matrix metalloproteinase inhibition with developing heart failure: comparative effects on left ventricular function and geometry. J Pharmacol Exp Ther 1999; 291: 799-811.

36. Zhang X, Yang YJ, Zhu WL, Huang Y, Zhu ZM. [Effects of angiotensin II receptor antagonism on matrix metalloproteinases and fibronectin in rats with experimental myocardial infarction]. Zhonghua Xin Xue Guan Bing Za Zhi 2006; 34: 1029-34. (Chinese)

37. Rohde LE, Ducharme A, Arroyo LH, et al. Matrix metalloproteinase inhibition attenuates early left ventricular enlargement after experimental myocardial infarction in mice. Circulation 1999; 99: 3063-70.

38. Herskowitz A, Choi S, Ansari AA, Wesselingh S. Cytokine mRNA expression in postischemic/reperfused myocardium. Am J Pathol 1995; 146: 419-28.

39. Latini R, Bianchi M, Correale E, et al. Cytokines in acute myocardial infarction: selective increase in 
circulating tumor necrosis factor, its soluble receptor, and interleukin-1 receptor antagonist. J Cardiovasc Pharmacol 1994; 23: 1-6.

40. Elmas E, Lang S, Dempfle CE, et al. High plasma levels of tissue inhibitor of metalloproteinase-1 (TIMP-1) and interleukin-8 (IL-8) characterize patients prone to ventricular fibrillation complicating myocardial infarction. Clin Chem Lab Med 2007; 45: 1360-5. 